\title{
Convexity of Reflective Submanifolds in Special Unitary Groups
}

\author{
Felix PLATZER and Peter QUAST \\ Universität Augsburg \\ (Communicated by K. Ahara)
}

\begin{abstract}
A submanifold of a Riemannian manifold is called reflective, if it is a connected component of an involutive isometry. If every shortest geodesic arc of a complete submanifold is still shortest in the ambient space, we say that the submanifold is convex. In this note we show that reflective submanifolds in special unitary groups are convex.
\end{abstract}

\section{Introduction}

M. S. Tanaka and the second author have shown in [8, Theorem 1] that every reflective submanifold of a symmetric $R$-space is convex. Since the unitary group

$$
\mathrm{U}_{n}:=\left\{A \in \mathbf{C}^{n \times n}: A \bar{A}^{T}=I\right\}
$$

endowed with the bi-invariant Riemannian metric induced by the scalar product $\langle X, Y\rangle=$ $-\operatorname{trace}(X Y)$ on its Lie algebra $\mathfrak{u}_{n}=\left\{A \in \mathbf{C}^{n \times n}: A=-\bar{A}^{T}\right\}$ is a symmetric $R$-space (see e.g. [3] and [1, pp. 310-311]), we get immediately:

PROPOSITION 1. Every reflective submanifold of a unitary group is convex.

On the other hand, special unitary groups

$$
\mathrm{SU}_{n}=\left\{A \in \mathrm{U}_{n}: \operatorname{det}(A)=1\right\} \subset \mathrm{U}_{n}
$$

with $n \geq 3$ endowed with the trace metric described above are prominent examples of compact symmetric spaces that are not $R$-spaces. Moreover $\mathrm{SU}_{n}, n \geq 3$, is not a convex submanifold of $\mathrm{U}_{n}$. One might therefore wonder if the statement of Proposition 1 is still true for special unitary groups. In this note we give an affirmative answer:

THEOREM 1. Every reflective submanifold of a special unitary group is convex.

Received November 6, 2013; revised July 15, 2014

2010 Mathematics Subject Classification: 51F25 (Primary), 53C40 (Secondary)

Key words and phrases: special unitary groups, convexity, reflective submanifolds 
Note that Theorem 1 does not extend to reflective submanifolds of arbitrary compact Lie groups with bi-invariant Riemannian metrics, as the example of the center-free group $\mathrm{SU}_{n} / \mathbf{Z}_{n}, n \geq 3$, shows (see [8, Section 4]).

The results presented here are part of the first author's exam project under the supervision of the second author. The authors wish to thank J.-H. Eschenburg and E. Heintze for helpful remarks.

\section{Preliminaries}

A reflective submanifold of a Riemannian manifold $M$ is a connected component of the fixed point set of an involutive isometry $\tau$ of $M$, that is an isometry $\tau$ of $M$ with $\tau \neq \mathrm{id}$ and $\tau \circ \tau=$ id. In particular, reflective submanifolds are totally geodesic (see e.g. [1, Proposition 8.3.4]) and closed submanifolds. Thus, if $M$ is compact then any reflective submanifold of $M$ is compact, too.

A connected submanifold $S \subset M$ of a Riemannian manifold is called (geodesically) convex, if for any two points $x, y \in S$ the Riemannian distance (see e.g. [11, p. 26] for the definition) $d_{S}(x, y)$ measured within $S$ coincides with the Riemannian distance $d_{M}(x, y)$ measured within the ambient space $M$. A complete submanifold $S \subset M$ is convex, if any shortest geodesic segment in $S$ is still shortest in $M$.

The tangent cut locus $C\left(T_{o} M\right)$ of a complete Riemannian manifold $M$ at a point $o \in M$ is the set formed by all $X \in T_{o} M$ such that the geodesic ray $\gamma_{X}$ in $M$ that starts at $o$ in direction $X$ satisfies

- $d_{M}\left(o, \gamma_{X}(t)\right)=t\|X\|$ for $t \in[0,1]$ and

- $d_{M}\left(o, \gamma_{X}(t)\right)<t\|X\|$ for $t>1$.

The tangent cut locus $C\left(T_{o} M\right)$ is star-shaped with center $0 \in T_{o} M$. Further details about the tangent cut locus can, for example, be found in [11]. We observe that a compact submanifold $S \subset M$ is convex, if and only if

$$
C\left(T_{o} S\right)=C\left(T_{o} M\right) \cap T_{o} S
$$

holds for any point $o \in S$. Adapting a result of Sakai (see [9]) to submanifolds, Tasaki showed:

PROPOSITION 2 ([12, Lemma 2.2]). Let M be a connected compact symmetric space and let $S \subset M$ be a compact connected totally geodesic submanifold of $M$. Let $A \subset S$ be a maximal flat totally geodesic torus in $S$ and $\tilde{A} \subset M$ a maximal flat totally geodesic torus in $M$ that contains $A$. We choose a point $o \in A$. If

$$
C\left(T_{o} A\right)=C\left(T_{o} \tilde{A}\right) \cap T_{o} A,
$$

then $S$ is a convex submanifold of $M$.

REMARK 1. In the hypothesis of [12, Lemma 2.2], Tasaki assumes that $M=G / K$ and $S=L / H$ for Riemannian symmetric pairs $(G, K)$ and $(L, H)$ with $L \subset G$ and $H \subset K$. 
In the above context one may take as $G$ the identity component of the isometry group of $M$ and set $K:=\{g \in G: g(o)=o\}$ for a chosen point $o \in S \subset M$. Then $L$ denotes the identity component of $\{g \in G: g(S) \subset S\}$ and $H:=\{l \in L: l(o)=o\}$ (see [8, Observation 3] for details).

Recall that the rank of a compact symmetric space $M$, denoted by $\operatorname{rank}(M)$, is the dimension of a maximal flat totally geodesic torus in $M$. As a direct consequence of Proposition 2 we get:

COROLLARY 1. Let $S$ be a compact connected totally geodesic submanifold of a connected compact symmetric space $M$ with $\operatorname{rank}(S)=\operatorname{rank}(M)$. Then any maximal flat totally geodesic torus in $S$ is also a maximal flat totally geodesic torus in $M$, and $S$ is convex in $M$.

In particular, any compact connected totally geodesic submanifold $S$ of $\mathrm{SU}_{n}, n \geq 2$, with $\operatorname{rank}(S)=\operatorname{rank}\left(\mathrm{SU}_{n}\right)=n-1$ is convex in $\mathrm{SU}_{n}$.

\section{Proof of the main result}

In this section we prove the main result of this note, Theorem 1. Since $\mathrm{SU}_{2}$ is isometric to a standard 3-sphere and hence a symmetric $R$-space, we only need to consider the case $\mathrm{SU}_{n}$ with $n \geq 3$ in view of [8, Theorem 1].

The full isometry group $\mathrm{Iso}\left(\mathrm{SU}_{n}\right)$ acts by conjugation on the set

$$
\operatorname{Inv}\left(\mathrm{SU}_{n}\right):=\left\{\tau \in \operatorname{Iso}\left(\mathrm{SU}_{n}\right): \tau \neq \mathrm{id}, \tau \circ \tau=\mathrm{id}\right\}
$$

of all involutive isometries of $\mathrm{SU}_{n}$. For an involutive isometry $\tau \in \operatorname{Inv}\left(\mathrm{SU}_{n}\right)$ we denote by $\operatorname{Fix}(\tau):=\left\{A \in \mathrm{SU}_{n}: \tau(A)=A\right\}$ the set of all fixed points of $\tau$. If $X \in \operatorname{Fix}(\tau)$ then $\operatorname{Fix}_{X}(\tau)$ designates the connected component of Fix $(\tau)$ that contains $X$. For $g \in \operatorname{Iso}\left(\operatorname{SU}_{n}\right)$ we obviously have

$$
\operatorname{Fix}\left(g \circ \tau \circ g^{-1}\right)=g(\operatorname{Fix}(\tau)) .
$$

ObSERVATION 1. Let $\tau \in \operatorname{Inv}\left(\operatorname{SU}_{n}\right)$ and let $X \in \operatorname{Fix}(\tau)$, then

$$
l_{X^{-1}}\left(\operatorname{Fix}_{X}(\tau)\right)=\operatorname{Fix}_{I}\left(l_{X^{-1}} \circ \tau \circ l_{X}\right),
$$

where $l_{X}$ denotes left multiplication with $X$ and $I$ the identity matrix.

Since isometries preserve convexity, we only need to look at $\operatorname{Fix}_{I}(\tau)$ for any $\tau \in$ $\operatorname{Inv}\left(\mathrm{SU}_{n}\right)$, in order to prove Theorem 1. Involutive isometries of $\mathrm{SU}_{n}$ that fix the identity $I \in \mathrm{SU}_{n}$ can be described as follows:

LEMMA 1 (see e.g. [5, proof of Theorem 3.3] and [7, Proposition C.1]). Let $\tau$ be an isometry of $\mathrm{SU}_{n}$ that fixes I and satisfies $\tau \circ \tau=\mathrm{id}$. Then either $\tau$ or $\iota \circ \tau$ is a Lie group automorphism of $\mathrm{SU}_{n}$ that squares to the identity, where เ denotes the inversion $\iota(A)=A^{-1}=\bar{A}^{T}$ on $\mathrm{SU}_{n}$. 
Moreover, if $\iota$ $\tau$ is a Lie group automorphism, then $\operatorname{Fix}_{I}(\tau)$ is a totally geodesic submanifold of $\mathrm{SU}_{n}$ isometric (up to an appropriate scaling) to the irreducible symmetric space $\mathrm{SU}_{n} / \mathrm{Fix}(\iota \circ \tau)$.

From the classification of compact irreducible symmetric spaces, originally due to Élie Cartan in the 1920th, we know that up to conjugation with Lie group automorphisms there are only three kinds of involutive Lie group automorphisms of special unitary groups (see e.g. [2, pp. 451-452]), namely:

$$
\begin{aligned}
\tau_{1, p}: & \mathrm{SU}_{n} \rightarrow \mathrm{SU}_{n}, A \mapsto I_{p, q} A I_{p, q}^{-1}, \text { with } I_{p, q}:=\left(\begin{array}{ll}
I_{p} & \\
& -I_{q}
\end{array}\right) \\
& \text { for } 1 \leq p \leq n-1 \text { and } q=n-p ; \\
\tau_{2}: & \mathrm{SU}_{n} \rightarrow \mathrm{SU}_{n}, A \mapsto \bar{A} ; \\
\tau_{3}: & \mathrm{SU}_{2 n} \rightarrow \mathrm{SU}_{2 n}, A \mapsto J \bar{A} J^{-1}, \text { with } J=\left(\begin{array}{cc} 
& -I_{n} \\
I_{n} &
\end{array}\right) .
\end{aligned}
$$

We have

$$
\begin{aligned}
& \operatorname{Fix}_{I}\left(\tau_{1, p}\right)=S\left(\mathrm{U}_{p} \times \mathrm{U}_{n-p}\right)=\left\{\left(\begin{array}{ll}
A & \\
& B
\end{array}\right) \in \mathrm{SU}_{n}: A \in \mathrm{U}_{p}, B \in \mathrm{U}_{n-p}\right\}, \\
& \operatorname{Fix}_{I}\left(\tau_{2}\right)=\mathrm{SO}_{n}=\mathrm{SU}_{n} \cap \mathbf{R}^{n \times n}, \\
& \operatorname{Fix}_{I}\left(\tau_{3}\right)=\mathrm{Sp}_{n}=\left\{\left(\begin{array}{cc}
A & B \\
-\bar{B} & \bar{A}
\end{array}\right) \in \mathrm{SU}_{2 n}: A, B \in \mathbf{C}^{n \times n}\right\} .
\end{aligned}
$$

With Lemma 1 we conclude that besides $\tau_{1, p}, \tau_{2}$ and $\tau_{3}$ there are up to conjugation with Lie group automorphisms three further types of involutive isometries (which are group antiautomorphisms) of special unitary groups, namely:

$$
\begin{array}{rlll}
\tau_{4, p}:=\iota \circ \tau_{1, p}: & \mathrm{SU}_{n} \rightarrow \mathrm{SU}_{n}, & A \mapsto I_{p, q} A^{-1} I_{p, q}^{-1}, \quad 0 \leq p \leq n-1, \\
\tau_{5}:=\iota \circ \tau_{2}: & \mathrm{SU}_{n} \rightarrow \mathrm{SU}_{n}, & A \mapsto A^{T}, \\
\tau_{6}:=\iota \circ \tau_{3}: & \mathrm{SU}_{2 n} \rightarrow \mathrm{SU}_{2 n}, & A \mapsto J A^{T} J^{T} . &
\end{array}
$$

with

$$
\begin{aligned}
& \operatorname{Fix}_{I}\left(\tau_{4, p}\right) \cong \mathrm{SU}_{n} / S\left(\mathrm{U}_{p} \times \mathrm{U}_{n-p}\right), \\
& \operatorname{Fix}_{I}\left(\tau_{5}\right) \cong \mathrm{SU}_{n} / \mathrm{SO}_{n}, \\
& \operatorname{Fix}_{I}\left(\tau_{6}\right) \cong \mathrm{SU}_{2 n} / \mathrm{Sp}_{n} .
\end{aligned}
$$

Reflective submanifolds in symmetric spaces have been classified by Leung in a series of papers $[4,5,6]$. Here we just recovered the case $\mathrm{SU}_{n}$ in [5, Theorem 3.3].

Notice that any of the above given involutive isometries $\tau$ of a special unitary group extends canonically to an involutive isometry $\tilde{\tau}$ of the corresponding unitary group, where $\tilde{\tau}$ is defined by the same formula as $\tau$. With Proposition 1 we get: 
OBSERVATION 2. Let $\tau$ be an involutive isometry of $\mathrm{SU}_{n}$ that extends canonically to an involutive isometry $\tilde{\tau}$ of $\mathrm{U}_{n}$. If $\operatorname{Fix}_{I}(\tilde{\tau})$ is contained in $\mathrm{SU}_{n}$, then $\operatorname{Fix}_{I}(\tau)$ is convex in $\mathrm{SU}_{n}$.

Since the determinant of a fixed point of $\tilde{\tau}_{2}, \tilde{\tau}_{3}$ or $\tilde{\tau}_{4, p}$ is either 1 or -1 , we conclude:

LEMmA 2. The submanifolds $\operatorname{Fix}_{I}\left(\tau_{2}\right), \operatorname{Fix}_{I}\left(\tau_{3}\right)$ and $\operatorname{Fix}_{I}\left(\tau_{4, p}\right)$ are convex.

With $\operatorname{rank}\left(S\left(\mathrm{U}_{p} \times \mathrm{U}_{n-p}\right)\right)=\operatorname{rank}\left(\mathrm{SU}_{n} / \mathrm{SO}_{n}\right)=n-1$ (see e.g. [2, Chapter X]), we get by Corollary 1 :

LEMmA 3. The submanifolds $\operatorname{Fix}_{I}\left(\tau_{1, p}\right)$ and $\operatorname{Fix}_{I}\left(\tau_{5}\right)$ are convex.

To complete the proof of Theorem 1 it remains to be shown:

LEMMA 4. The submanifold $S:=\operatorname{Fix}_{I}\left(\tau_{6}\right)$ of $\mathrm{SU}_{2 n}, n \geq 2$, is convex.

PROOF. Notice that $S:=\operatorname{Fix}_{I}\left(\tau_{6}\right)$ can be identified with $\mathrm{SU}_{2 n} / \mathrm{Sp}_{n}$, by the following transitive group action

$$
\mathrm{SU}_{2 n} \times S \rightarrow S, \quad(A, X) \mapsto A X J A^{T} J^{T},
$$

where $\mathrm{Sp}_{n}$ is the stabilizer of the identity.

Since $\tau_{6}$ is the restriction of the linear involution

$$
\hat{\tau}_{6}: \mathbf{C}^{2 n \times 2 n} \rightarrow \mathbf{C}^{2 n \times 2 n}, \quad A \mapsto J A^{T} J^{T},
$$

the tangent space $T_{I} S$ can be written as

$$
\mathfrak{s}:=T_{I} S=\mathfrak{s u}_{2 n} \cap \operatorname{Fix}\left(\hat{\tau}_{6}\right)=\left\{\left(\begin{array}{cc}
A & B \\
B & A^{T}
\end{array}\right): A \in \mathfrak{s u}_{n}, B \in \mathbf{C}^{n \times n}, B=-B^{T}\right\}
$$

(see also e.g. [2, p. 452, type AII]). Recall that $\operatorname{rank}(S)=\operatorname{rank}\left(\mathrm{SU}_{2 n} / \mathrm{Sp}_{n}\right)=n-1$ (see e. g. [2, p. 518]). We choose a maximal abelian subspace $\mathfrak{a}$ of $\mathfrak{s}$ as follows

$$
\mathfrak{a}:=\left\{\left(\begin{array}{cc}
D & 0 \\
0 & D
\end{array}\right): D \in\left(i \cdot \mathbf{R}^{n \times n}\right) \cap \operatorname{Diag}_{n}, \operatorname{trace}(D)=0\right\},
$$

where $\operatorname{Diag}_{n}$ is the set of all diagonal matrices in $\mathbf{C}^{n \times n}$. It can be extended to the maximal abelian subspace

$$
\tilde{\mathfrak{a}}:=\left\{\tilde{D} \in\left(i \cdot \mathbf{R}^{2 n \times 2 n}\right) \cap \operatorname{Diag}_{2 n}: \operatorname{trace}(\tilde{D})=0\right\}
$$

of $\mathfrak{s u}_{2 n} \cong T_{I} \mathrm{SU}_{2 n}$. The Dynkin diagram of $\mathrm{SU}_{2 n}$ is of type $\mathfrak{a}_{2 n-1}$ (see e.g. [2, p. 516]), and we choose a fundamental basis $\left\{\tilde{\alpha}_{1}, \ldots, \tilde{\alpha}_{2 n-1}\right\}$ of the root system of $\mathrm{SU}_{2 n}$ corresponding to $\tilde{\mathfrak{a}}$ as follows:

$$
\begin{aligned}
\tilde{\alpha}_{j} & :=\varepsilon_{j}-\varepsilon_{n+j}, \quad j=1, \ldots, n ; \\
\tilde{\alpha}_{n+l} & :=\varepsilon_{n+l}-\varepsilon_{l+1}, \quad l=1, \ldots, n-1,
\end{aligned}
$$


with

$$
\varepsilon_{k}: \tilde{\mathfrak{a}} \rightarrow \mathbf{R}, \quad i\left(\begin{array}{ccc}
x_{1} & & \\
& \ddots & \\
& & x_{2 n}
\end{array}\right) \mapsto x_{k}
$$

for $k=1, \ldots, 2 n$ and associated highest root

$$
\tilde{\delta}=\sum_{j=1}^{2 n-1} \tilde{\alpha}_{j}=\varepsilon_{1}-\varepsilon_{2 n}
$$

(see e.g. [2, pp. 462, 476]). The corresponding Weyl chamber of $\mathfrak{s u}_{2 n}$ in $\tilde{\mathfrak{a}}$ is

$$
\tilde{\mathfrak{a}}^{+}=\left\{X \in \tilde{\mathfrak{a}}: \tilde{\alpha}_{j}(X)>0 \text { for all } j=1, \ldots, 2 n-1\right\} .
$$

The Dynkin diagram of the symmetric space $S$ has type $\mathfrak{a}_{n-1}$ (see e.g. [2, Chapter X]). Setting

$$
\alpha_{j}:=\left.\tilde{\alpha}_{n+j}\right|_{\mathfrak{a}}
$$

for $j=1, \ldots, n-1$ we get a fundamental basis $\left\{\alpha_{1}, \ldots, \alpha_{n-1}\right\}$ of the root system of $S$ corresponding to $\mathfrak{a}$ with highest root

$$
\delta=\sum_{j=1}^{n-1} \alpha_{j}=\left.\varepsilon_{1}\right|_{\mathfrak{a}}-\left.\varepsilon_{n}\right|_{\mathfrak{a}}
$$

The closure $\overline{\mathfrak{a}^{+}}$of the corresponding Weyl chamber

$$
\mathfrak{a}^{+}:=\left\{X \in \mathfrak{a}: \alpha_{j}(X)>0 \text { for all } j=1, \ldots, n-1\right\}
$$

in $\mathfrak{a}$ is contained in the closure $\overline{\tilde{\mathfrak{a}}^{+}}$of $\tilde{\mathfrak{a}}^{+}$.

Since every element of $\mathfrak{a}$ can be mapped into $\overline{\mathfrak{a}^{+}}$by conjugation with an element of $\mathrm{Sp}_{n}$ (linear isotroy action of $S$ ), the tangent cut locus $C(\mathfrak{a})=C\left(T_{I} A\right)$ of the maximal flat totally geodesic torus $A:=\exp (\mathfrak{a})$ in $S$ is already determined by $C(\mathfrak{a}) \cap \overline{\mathfrak{a}^{+}}$(see [10]). Similarly, every element of $\tilde{\mathfrak{a}}$ is conjugate to an element of $\overline{\tilde{\mathfrak{a}}^{+}}$by an element of $\mathrm{SU}_{2 n}$. Thus, to determine the tangent cut locus $C(\tilde{\mathfrak{a}})=C\left(T_{I} \tilde{A}\right)$ of the maximal flat totally geodesic torus $\tilde{A}:=\exp (\tilde{\mathfrak{a}})$ in $\mathrm{SU}_{2 n}$, it is sufficient to know $C(\tilde{\mathfrak{a}}) \cap \overline{\tilde{\mathfrak{a}}^{+}}$. Following Sakai (see [10, p. 198]) we get

$$
C(\mathfrak{a}) \cap \overline{\mathfrak{a}^{+}}=\left\{X \in \overline{\mathfrak{a}^{+}}: \delta(X)=2 \pi\right\} \quad \text { and } \quad C(\tilde{\mathfrak{a}}) \cap \overline{\tilde{\mathfrak{a}}^{+}}=\left\{X \in \overline{\tilde{\mathfrak{a}}^{+}}: \tilde{\delta}(X)=2 \pi\right\} .
$$

Let $X=\left(\begin{array}{ll}D & \\ & D\end{array}\right)$ with $D=i\left(\begin{array}{ccc}x_{1} & & \\ & \ddots & \\ & & x_{n}\end{array}\right), x_{1}, \ldots, x_{n} \in \mathbf{R}$, be an element of $\overline{\mathfrak{a}^{+}}$, that 
is $x_{1} \geq x_{2} \geq \cdots \geq x_{n}$ with $\sum_{j=1}^{n} x_{j}=0$. Then $X$ lies in $C(\mathfrak{a})$ if and only if

$$
\delta(X)=x_{1}-x_{n}=2 \pi .
$$

But in this case we also have $\tilde{\delta}(X)=2 \pi$, that is $X \in C(\tilde{\mathfrak{a}})$. We conclude that

$$
C(\mathfrak{a}) \cap \overline{\mathfrak{a}^{+}}=C(\tilde{\mathfrak{a}}) \cap \overline{\mathfrak{a}^{+}}
$$

and therefore

$$
C(\mathfrak{a})=C(\tilde{\mathfrak{a}}) \cap \mathfrak{a} .
$$

Lemma 4 now follows from Proposition 2.

\section{References}

[1] J. Berndt, S. Console and C. Olmos, Submanifolds and holonomy, Chapman Hall/CRC Res. Notes in Math. 434, Chapman \& Hall/CRC, Boca Raton, 2003.

[2] S. Helgason, Differential geometry, Lie groups and symmetric spaces, Pure Appl. Math. 80, Academic Press, New York, 1978.

[ 3 ] S. Kobayashi and T. Nagano, On filtered Lie algebras and geometric structures I, J. Math. Mech. 13 (1964), 875-907.

[4] D. S. P. LEUNG The reflection principle for minimal submanifolds of Riemannian symmetric spaces, J. Differential Geom. 8 (1973), 153-160.

[ 5 ] D.S.P. LEUNG, On the classification of reflective submanifolds of Riemannian symmetric spaces, Indiana Univ. Math. J. 24 (1974), 327-339.

[ 6 ] D.S.P. LEUNG, Reflective submanifolds. III. Congruency of isometric reflective submanifolds and corrigenda to the classification of reflective submanifolds, J. Differential Geom. 14 (1979), 167-177.

[ 7 ] A.-L. MARE and P. QuAst, Bott periodicity for inclusions of symmetric spaces, Doc. Math., J. DMV 17 (2012), 911-952.

[ 8 ] P. QUAST and M. S. TANAKA, Convexity of reflective submanifolds in symmetric R-spaces, Tohoku Math. J. (2) 64 (2012), 607-616.

[9] T. SAKAI, On cut loci of compact symmetric spaces, Hokkaido Math. J. 6 (1977), 136-161.

[10] T. SAKAI, Cut loci of compact symmetric spaces, Minimal submanifolds and geodesics (Proc. Japan-United States Sem., Tokyo, 1977) Kaigai Publications, Tokyo 1978, 193-207.

[11] T. SAKAI, Riemannian Geometry, Translations of Mathematical Monographs. 149, American Mathematical Society, Providence 1996.

[12] H. TASAKI, The intersection of two real forms in the complex hyperquadric, Tohoku Math. J. (2) 62 (2010), 375-382. 
Present Addresses:

FELIX Platzer

INSTITUT FÜR MATHEMATIK,

UNIVERSITÄT AUgSBURG,

D 86135 Augsburg, Germany.

e-mail: felix@platzer-riederau.de

PETER QuAst

INSTITUT FÜR MATHEMATIK,

UNIVERSITÄT AUGSBURG,

D 86135 Augsburg, Germany.

e-mail: peter.quast@math.uni-augsburg.de 